\title{
A Numerical Optimization Approach For Tuning Fuzzy Logic Controllers
}

\author{
Stanley E. Woodard \\ NASA Langley Research Center
}

Devendra P. Garg

Duke University

\begin{abstract}
This paper develops a method to tune fuzzy controllers using numerical optimization. The main attribute of this approach is that it allows fuzzy logic controllers to be tuned to achieve global performance requirements. Furthermore, this approach allows design constraints to be implemented during the tuning process. The method tunes the controller by parameterizing the membership functions for error, change-in-error and control output. The resulting parameters form a design vector which is iteratively changed to minimize an objective function. The minimal objective function results in an optimal performance of the system. A spacecraft mounted science instrument line-of-sight pointing control is used to demonstrate results.
\end{abstract}




\section{Introduction}

Fuzzy logic control extends fuzzy set theory to the control of processes [1-5]. A fuzzy logic controller (FLC) is a fuzzy expert system which uses approximate reasoning. To date, almost all FLCs use only "if-then" rules. The basic scheme of using FLC for feedback error control is to generate a control input based upon error information resulting from feedback $[5-7,2]$. The control input to the system is a desired or referenced input. Conceptually, FLC is similar to traditional feedback control except that the fuzzy controller requires the user to formulate membership functions for various fuzzy sets, develop rules that map input conditions to responses using fuzzy sets, and select fuzzification and defuzzification techniques for a small number of options.

The basic architecture for fuzzy logic control is depicted in Fig. 1. As shown in Fig. 1, outputs from the controlled system are inputs to a fuzzification process. This fuzzification process maps the inputs to membership in fuzzy sets. Fuzzification relates vague and imprecise inputs to approximate reasoning. Following the fuzzification process is decision making logic where control action is predicated from rules of inference. The inference outputs are fuzzy. A single crisp control output is produced in a defuzzification process. Control is predicated upon a measured error and change-in-error. As an example, if error is positive and small, and change-in-error is zero then control output is negative and small. Ref. 5 gives a detailed description of the application of fuzzy logic control.

The advantage of using fuzzy controllers is that they have been shown to produce very good results in cases where the mathematical description of the system being controlled 
may not be readily available or the description may be of questionable fidelity [8-13]. Despite the use of simple fuzzy logic control, there are specific drawbacks $[12,14]$ due to tuning the controllers to meet some performance objective.

Methods for tuning fuzzy controllers include using neural networks, fuzzy self-organizing control (SOC), genetic algorithms, and human knowledge. The SOC is essentially a decision maker predicated upon performance feedback. The SOC is capable of generating and modifying the control rules based upon an evaluation of their performance. The SOC is composed of three elements: performance index evaluation, credit assignment, and rule modification. The performance index and credit assignment require a priori knowledge of desired controller input-output mapping. Daley and Gill $[12,14]$ have demonstrated the use of SOC for the attitude control of a spacecraft.

Neural Networks have also been used because of their learning capabilities for system identification and/or tuning. However, simultaneous tuning of membership functions and identification of inference rules is difficult. Rules identified by networks are difficult to understand. Furthermore, training neural networks is computationally time consuming. Genetic algorithms are a form of directed random search. To implement genetic algorithms, the fuzzy sets must be parameterize discretely. The discrete set forms an initial population for the genetic algorithm. The population evolves to produce new, but hopefully better design of the fuzzy sets. Human knowledge can be used to tune the controllers. However, such knowledge must be developed into a reliable linguistic model of an operator's strategy. Another consideration for using user knowledge is that the system processes may change beyond the operator's realm of experience. 
This paper presents a new approach for tuning fuzzy logic controllers using numerical optimization. The main attribute of this approach is that it allows fuzzy logic controllers to be tuned to achieve global performance requirements. Furthermore, this approach allows design constraints to be implemented during the tuning process. The approach consists of specifying the desired outcome of tuning a fuzzy controller (e.g., system time response) as minimization of an objective function. The control design methodology is applied to a spacecraft mounted science instrument (payload) which rotates about a drive shaft. The design goal is to have a bounded response amplitude while having the instrument meet mission pointing requirements. Fuzzy logic control is used as a means to maintain either a constant slew rate or fixed line-of-sight pointing for the payload subjected to vibration due to multiple disturbance sources.

Design constraints such as physical limitations in hardware or software such as controller torque output (magnitude vs. frequency), measurement sampling rate, measurement sampling range (i.e., limits), etc. can be included in the optimization process and fuzzy logic controller development. Fuzzy membership support limits are naturally malleable to some system specifications such as error measurement range and control output. If prior knowledge of the controller's maximum output and measurement range (error and error rate) is available, a fuzzy logic controller can be tuned by having the control support limit as the controller's output maximum value. The error support limitation could be either the physical measurement limit or it could reflect a bound of measurement within a given regime of operation. The design methodology presented in this paper allows for constraints to be included in the tuning process either in the constraint equations or with the upper and lower bounds on the design vector. 


\section{Optimization Strategy - General}

An objective function is prescribed such that, as it is reduced in value, the overall performance improves. Furthermore, the objective must be explicitly or implicitly dependent upon a set of design parameters. Design parameters are given such that they can be varied to change the overall performance (design objective). Thus, the design objective is to minimize

$$
F(\alpha)
$$

subject to

$$
G_{j}(\alpha) \leq 0 \quad j=1, m
$$

and

$$
\alpha_{l_{i}} \leq \alpha_{i} \leq \alpha_{u_{i}} \quad i=1, n
$$

where $F(\alpha)$ is an objective function which, when minimized, will result in optimum performance of the system. The vector, $\alpha$, contains $n$ system parameters which are varied through the iterative optimization process. $G_{j}(\alpha)$ is the $j t h$ constraint on the design parameters. There are $m$ constraints. Each design parameter, $\alpha_{i}$, is bounded by upper and lower limits, $\alpha_{l_{i}}$ and $\alpha_{u_{i}}$, respectively.

A flowchart of the optimization procedure is shown in Fig. 2. The procedure is iterative. The first iteration requires the user to develop an optimization objective, constraint equations, and bounds for the elements of the design vector. In the first iteration, the objective function is evaluated. If the objective value is less than some desired value, the process is complete. If the objective value is greater than some desired value, the 
optimization process searches for new values for the elements of the design vector, $\alpha$, which will reduce the value of the objective function and satisfy the constraint equations. The permissible values for elements of $\alpha$ are those which are within the limits of the upper and lower bounds of $\alpha$. After the constraints are satisfied, the design vector is used to produce an objective function evaluation which is then compared to the desired objective value. The variation of the objective with respect to the change in the design vector is used to develop the next vector. Iteration of the optimization process is continued until some desirable value of $F(\alpha)$ is achieved or the number of iterations has reached some prescribed maximum.

\section{Controlled Payload}

The controlled payload mounted on the spacecraft is shown in Fig. 3. The payload equation of motion is

$$
I \ddot{\theta}=Q_{\theta}+u
$$

Payload rotation about the drive shaft is given by $\theta$. The spacecraft has two payloads. The disturbing payload is open-loop. The science instrument is the closed-loop controlled payload. The control torque, $u$, is the output from the defuzzification step of the fuzzy logic controller. The payload inertia, $I$, is $3.5 \mathrm{lb}-\mathrm{ft}^{2}$. Other external torques such as those due to damping are included in the term, $Q_{\theta}$. In Fig. 4 is shown the pointing control for the science instrument. The line-of-sight pointing is the combination of the instrument's elevation with respect to the spacecraft and the spacecraft's attitude. 


\section{Fuzzy Logic Control}

In Fig. 5 is shown a parameterized fuzzy membership function. The same membership functions are used for the error, change-in-error, and control output. The fuzzy term sets are positive big, (PB), positive small, (PS), zero, (Z), negative small, (NS), and negative big, (NB). These sets are symmetric about zero and are bounded by a parameter, $\alpha_{1}$, which defines minimum and maximum support limits. Positive small, (PS), and negative small, (NS), singletons (membership has a grade of 1.0) are located on the universe of discourse by $\alpha_{2}$. The base for the triangular membership function zero, $(\mathbf{Z})$, is defined by $\alpha_{3}$; negative small, (NS), and positive small, (PS), bases are defined by $\alpha_{4}$; and, the negative big, (NB), and positive big, (PB), bases are defined by $\alpha_{5}$. The initial membership functions (first optimization step) have an overlap at grade 0.65 . This overlap gives all support elements membership in two term sets unless the support element has membership grade of 1.0 in any term set. Because of the overlap, the control gradient with respect to the error and change-in-error is continuous, monotonic, and never zero [13]. The advantage this fuzzy membership offers is the effect that the membership functions have when the support elements are near zero. Unless the element is absolutely zero, the elements have nonzero grades on the respective side of zero. The result is a smooth approach to zero for process output as the support elements approach zero. The membership functions depicted in Fig. 5 are used for the payload error, change-in-error and control. The control rule matrix used is shown in Fig. 6. Each element of the matrix is numbered and corresponds to a rule. For example, Rule 7 states that if error is PS and change-in-error is NS then the control output should be $\mathbf{Z}$. 


\section{Optimization Strategy for Controller Design}

In this paper, the objective is derived from the system response. The optimization design objective, $F(\alpha)$, is to minimize the aggregate square of error between a measured position and commanded position. Thus

$$
F(\alpha)=\min \left[\sum_{t=0}^{t_{f}}\left(x(\alpha, t)-x_{c o m}\right)^{2}\right]
$$

where $x(\alpha, t)$ is the measured position at time $t$ and $x_{c o m}$ is the commanded position. The design vector, $\alpha$, has three sets of parameters, $\alpha_{1}, \ldots, \alpha_{5}$, for a total of 15 elements. Each membership function uses the following constraint to maintain the ordering of the set terms:

$$
G_{1}(\alpha)=1.25 \alpha_{2}-\alpha_{1}
$$

The following constraints assure continuous mapping from error and change-in-error to the control output. The constraints also assure overlap of adjacent membership functions. Because of the overlap, the control gradient with respect to the error and change-in-error is continuous, monotonic, and never zero. 


$$
\begin{gathered}
G_{2}(\alpha)=\left(\alpha_{1}-\alpha_{5}\right) * 1.10-\left(\alpha_{2}+\alpha_{4}\right) \\
G_{3}(\alpha)=-\alpha_{3}+\left(\alpha_{2}-\alpha_{4}\right) * 1.10
\end{gathered}
$$

A constraint is violated if

$$
G_{j}(\alpha)>0
$$

The flowchart for tuning the fuzzy controller is shown in Fig. 7. The general optimization procedure mentioned earlier is uesd. However, the design vector is used to generate the fuzzy logic membership functions. The system produces another response with the member functions. The new response is used to generate a new objective function evaluation.

The instrument fuzzy controller has three modes of operation: slew maneuvers, disturbance rejection (impulse and periodic vibration) and trajectory tracking. To demonstrate the methodology, the instrument controller will be tuned to perform slew maneuvers and disturbance rejection using the same initial design parameters. The initial design parameters were chosen such that they produce unsatisfactory system response. Successive iterations of the optimization process will reduce the objective function. The iterations terminate when a set of design parameters, $\alpha$, is generated which will produce a desired objective, $F(\alpha)$. 
Numerical optimization was performed using the Automated Design Synthesis (ADS) software [15]. The method of feasible directions for constrained minimization with sequential quadratic programming is used for optimization. In Figs. 8 through 10 are the initial and final membership functions for error, change-in-error, and control that were generated while tuning the instrument controller for a 0.5 radian slew maneuver. The membership functions for all term sets changed during the design process. However, changes for zero, $\mathbf{Z}$, were not as pronounced. The support limits for error, change-inerror and control changed from $0.1,0.01,0.01$ to $0.12,0.037,0.064$, respectively. An iteration history of the normalized objective (normalized to the objective evaluated during the first iteration) is shown in Fig. 11. The objective function was reduced to $1 \%$ of that evaluated for the first iteration after 60 iterations. The final (tuned) fuzzy controller produced a response which achieved the desired position in 23 sec. without overshoot, as shown in Fig. 12. After $40 \mathrm{sec}$, the response using the initial controller did not reach the desired commanded position of $0.5 \mathrm{rad}$.

In Figs. 13 and 14, are shown the results of tuning the instrument fuzzy controller while the instrument is commanded to maintain a position of $0.0 \mathrm{rad}$. in the presence of a periodic disturbance (amplitude $=0.01 \mathrm{ft}-1 \mathrm{~b}$ and frequency $=0.25 \mathrm{~Hz}$ ). The objective function, $F(\alpha)$, is reduced to $1 \%$ of the initial function after 27 iteration steps (Fig. 13). In Fig. 14 is shown the response for the initial and final controllers. The initial response amplitude is approximately $0.005 \mathrm{rad}$. The final response amplitude has' been reduced to approximately 0.00005 rad. 


\section{Tracking Controller Development}

The results presented thus far have shown the capability of tuning a fuzzy controller by parameterizing the membership functions and then using the parameters in a numerical optimization algorithm. The same initial membership functions were used in all cases. Reduction to $1 \%$ of initial objective function was used as the criterion for terminating the optimization process. In all cases, the final responses were satisfactory. The initial membership functions demonstrated the effectiveness of using numerical optimization to tune the controllers. The following discussion demonstrates that the approach can be used to enhance the tuning of controllers which already exhibit satisfactory performance.

A common need of many space-based payloads is the requirement to track a target, reposition, and track another target. Such retargeting occurs when communication antennae track relay satellites. The instrument controller was tuned by parametrically varying support limits for error, change-in-error, and, control output. The final design, arrived at in Ref. 16, is used as an initial design in the optimization process. The optimization design objective, $F(\alpha)$, is to minimize the aggregate square of error between a measured trajectory and desired trajectory. Thus

$$
F(\alpha)=\min \left[\sum_{t=0}^{t_{f}}\left(x(\alpha, t)-x_{d}(t)\right)^{2}\right]
$$

where $x_{d}(t)$ is the desired trajectory. After 71 design iterations, the objective function is reduced to $1 \%$ of its initial value. In Fig. 15a is shown the commanded elevation for the 
instrument to track. The results of using the initial and final designs are shown in Fig. 15b. The initial design has a tracking error greater than $0.25 \mathrm{rad}$. The final design of the fuzzy controller significantly tracks its commanded position better with a tracking error less than $0.01 \mathrm{rad}$.

\section{Concluding Remarks}

This paper has presented the development and viability of a method to tune fuzzy controllers using numerical optimization. A spacecraft science instrument pointing control model was used to demonstrate the application of the methodology. The optimization approach allows a designer to tune membership functions for all linguistic variables to achieve global performance. Furthermore, this approach allows design constraints to be implemented during the tuning process.

The optimization approach consists of specifying the desired outcome of tuning a fuzzy controller (e.g., system time response) as minimization of an objective function. The objective function is prescribed such that, as it is reduced in value, the overall performance improves. Furthermore, the objective must be explicitly or implicitly dependent upon a set of design parameters. Design parameters are given such that they can be varied to change the overall performance (design objective).

The controller was tuned by parameterizing the membership functions. The resulting parameters formed a design vector which was used in the iterative numerical optimization process. Optimization objectives used included minimizing the aggregate square of error between a measured position and commanded position; minimizing the aggregate absolute 
error between a measured position and commanded position; and minimizing the aggregate square of error between a measured trajectory and desired trajectory. In the design cases presented, the objective was reduced to $1 \%$ of its initial value within 80 optimization iterations.

Instrument design results included tuning the controller for 0.5 rad slew maneuver, disturbance rejection (impulse and periodic), and trajectory following. In tuning for the slew maneuver, the support limits for error, change-in-error, and control changed from $0.1,0.01,0.01$, to $0.12,0.037,0.064$, respectively. Using the final design, the instrument completed the maneuver in $23 \mathrm{sec}$. without overshoot. The final instrument controller design tuned for disturbance rejection when the instrument was subjected to a periodic disturbance resulted in a final response which reduced to 0.00005 rad (initial response was $0.005 \mathrm{rad}$ ). When subjected to unit impulse, the final instrument design was stable with a settling time of $21 \mathrm{sec}$. The final design used for tracking a trajectory reduced the tracking error to $0.01 \mathrm{rad}$ (initial tracking error was $0.25 \mathrm{rad}$ ). 


\section{References}

[1] Turksen, I. B., "Rule and Operation Decompositions in CRI," Advances in Fuzzy Theory and Technology, Edited by P. P. Wang, Bookwrights Press, Durham, North Carolina, 1993, pp. 219-256.

[2] Zadeh, L., "Fuzzy Logic," Computer, Computer Society of the IEEE, Vol. 21, April 1988, pp. 83-93.

[3] Smith, K. C., "Multiple-Valued Logic: A Tutorial and Appreciation," Computer, Computer Society of the IEEE, Vol. 21, April 1988, pp. 17-27.

[4] Aldridge, J., "Terminology and Concepts of Central and Fuzzy Logic," Proceedings of the AIAA Guidance, Navigation and Control Conference, New Orleans, Louisiana, August 12-14, 1991.

[5] Woodard, S. E., "Concurrent Fuzzy Logic Control of a Gimballed Payload and Space Spacecraft System," Ph.D. Paper, Dept. of Mechanical Engineering and Materials Science, Duke Univ., Durham, NC, Dec. 1995.

[6] Lee, C. C., "Fuzzy Logic in Control Systems: Fuzzy Logic Controller - Part 1," IEEE Transactions on Systems, Man and Cybernetics, Vol. 20, No. 2, March/April 1990, pp. 404-418.

[7] Lee, C. C., "Fuzzy Logic in Control Systems: Fuzzy Logic Controller - Part 2," IEEE Transactions on Systems, Man and Cybernetics, Vol. 20, No. 2, March/April 1990, pp. 419-435.

[8] Cunningham, G. B., Horstkotte, E. A., and Bochsler, D. C., "Integrating Fuzzy Logic Technology into Control Systems," Proceedings of the AIAA Guidance, Navigation and Control Conference, New Orleans, Louisiana, August 12-14, 1991, pp. 1699-1702.

[9] Schwartz, D. G., "Japanese Advances in Fuzzy Systems and Case-Based Reasoning," National Technical Information Service, No. PB92-115443, November 1991.

[10] Hirota, K., "Research Activities and Industrial Applications of Fuzzy Technology in Japan," Advances in Fuzzy Theory and Technology, Edited by P. P. Wang, Bookwwrights Press, Durham, North Carolina, 1993, pp. 269-282.

[11] Lea, R. N. and Jani, Y., "Fuzzy Logic in Autonomous Orbital Operations," Proceedings of the Second Joint Technology Workshop on Neural Networks and Fuzzy Logic, Volume 2, NASA Conference Publication 10061, February 1991, pp. 81-110.

[12] Daley, S. and Gill, K. F., "Comparison of a Fuzzy Logic Controller with a P + D Control Law," Journal of Dynamic Systems, Measurements, and Control, Vol. 111, June 1989, pp. 128-137. 
[13] Aldrige, J., Yashvant, J., and Zafar, T., "Tuning Fuzzy Controllers for Electric Motors," Proceedings of the Third International Symposium on Measurement and Controls in Robotics, Torino, Italy, September 21-24, 1993, pp. I-49 to I-53.

[14] Daley, S. and Gill, K. F., "Attitude Control of a Spacecraft Using an Extended SelfOrganizing Fuzzy Logic Controller," Proceedings of the Institution of Mechanical Engineers, Part C, Vol. 201, No. C2, 1987, pp. 97-106.

[15] Vanderplaats, G. N., "Automated Design Synthesis (ADS) - A FORTRAN Program for Automated Design Synthesis," Version 1.10, NASA CR 177985, September 1985.

[16] Woodard, S. E., Garg, D. P., Tyan, C. Y., and Wang, P. P., " An Application of Fuzzy Logic Control to a Gimballed Payload on a Space Spacecraft," Journal of Information Sciences- Applications, November 1995, Vol. 4, No. 3. pp 143-166. 


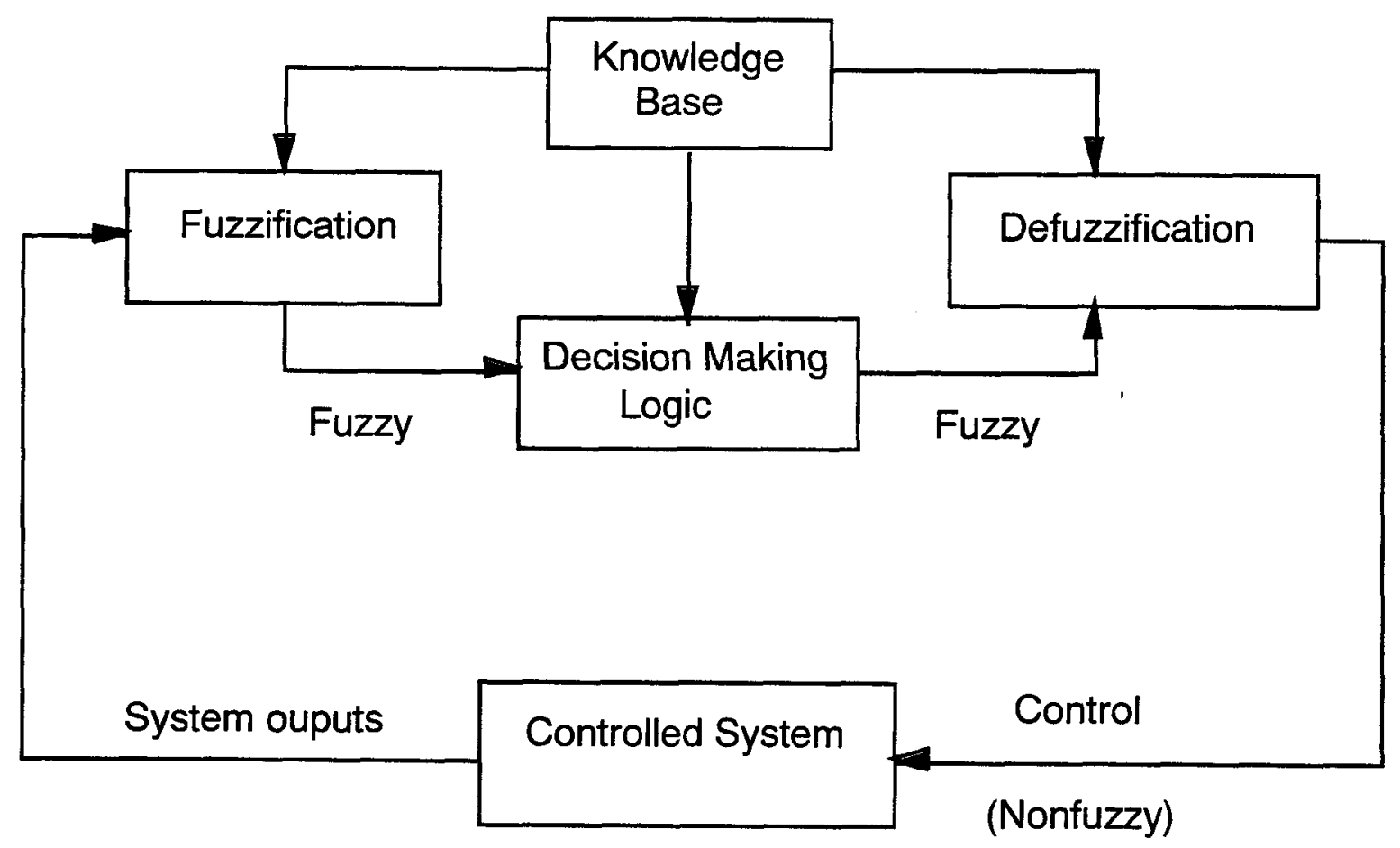

Fig.1 Schematic representation of fuzzy logic control. 


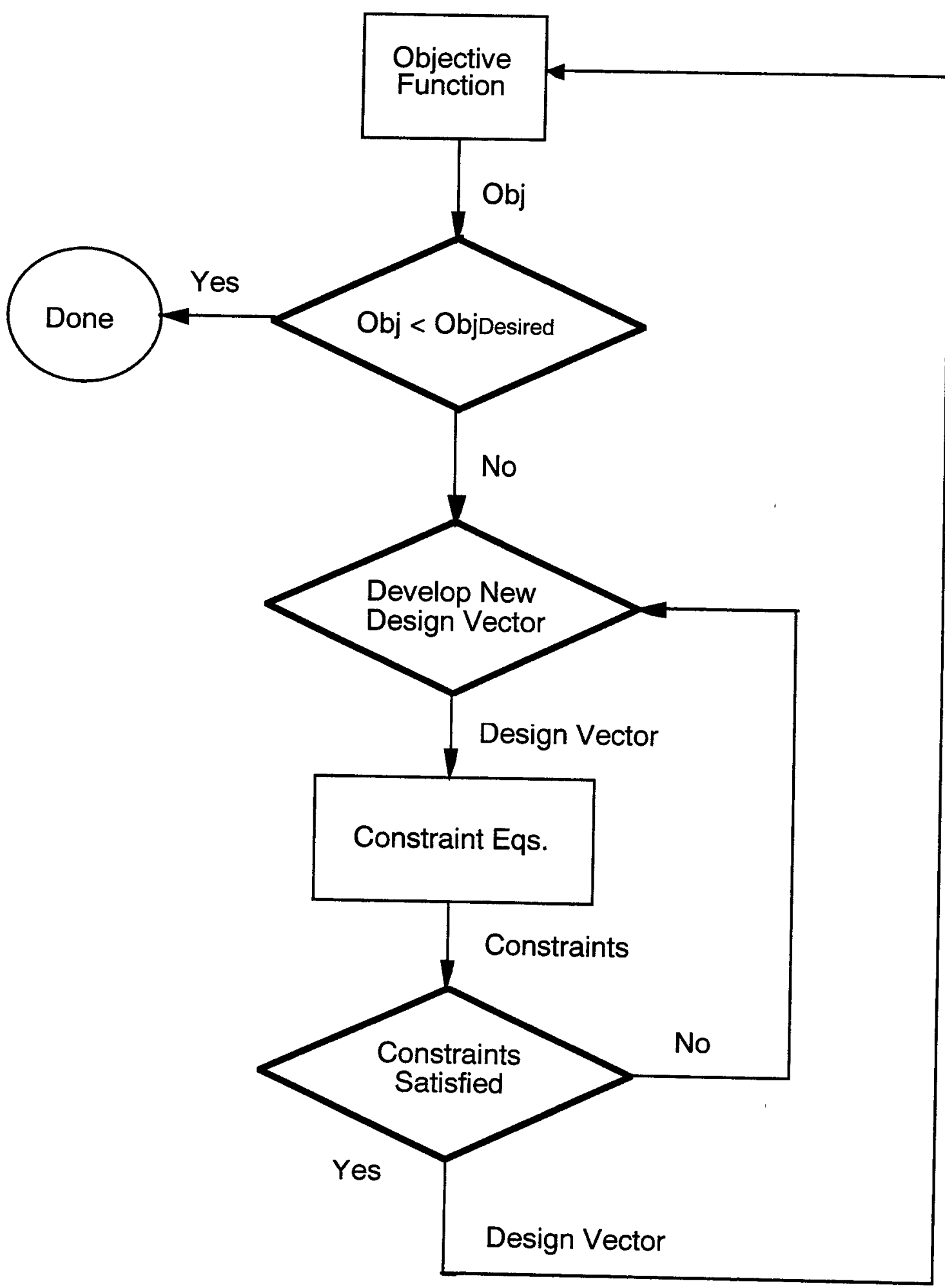

Fig. 2 Flowchart for general optimization. 


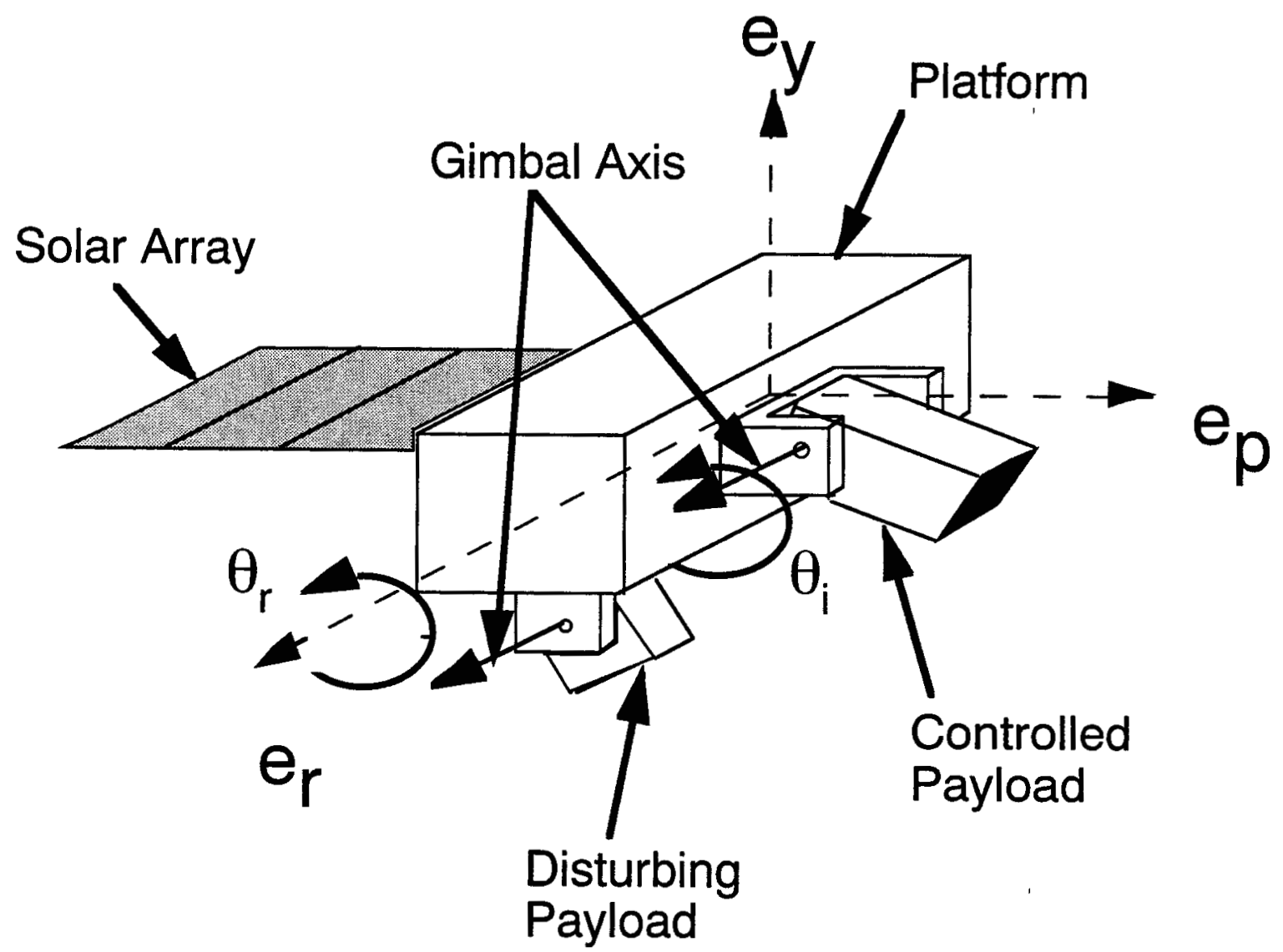

Fig. 3 Platform and payload System 


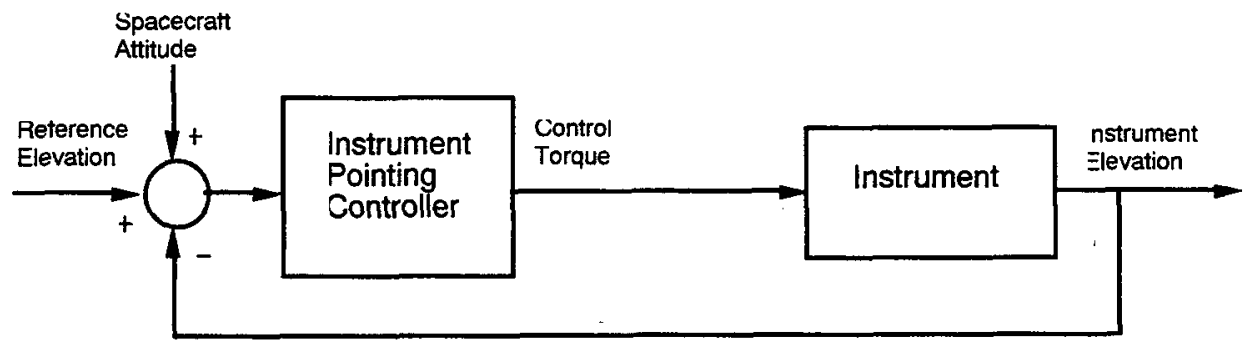

Fig. 4 Spacecraft mounted instrument pointing control. 


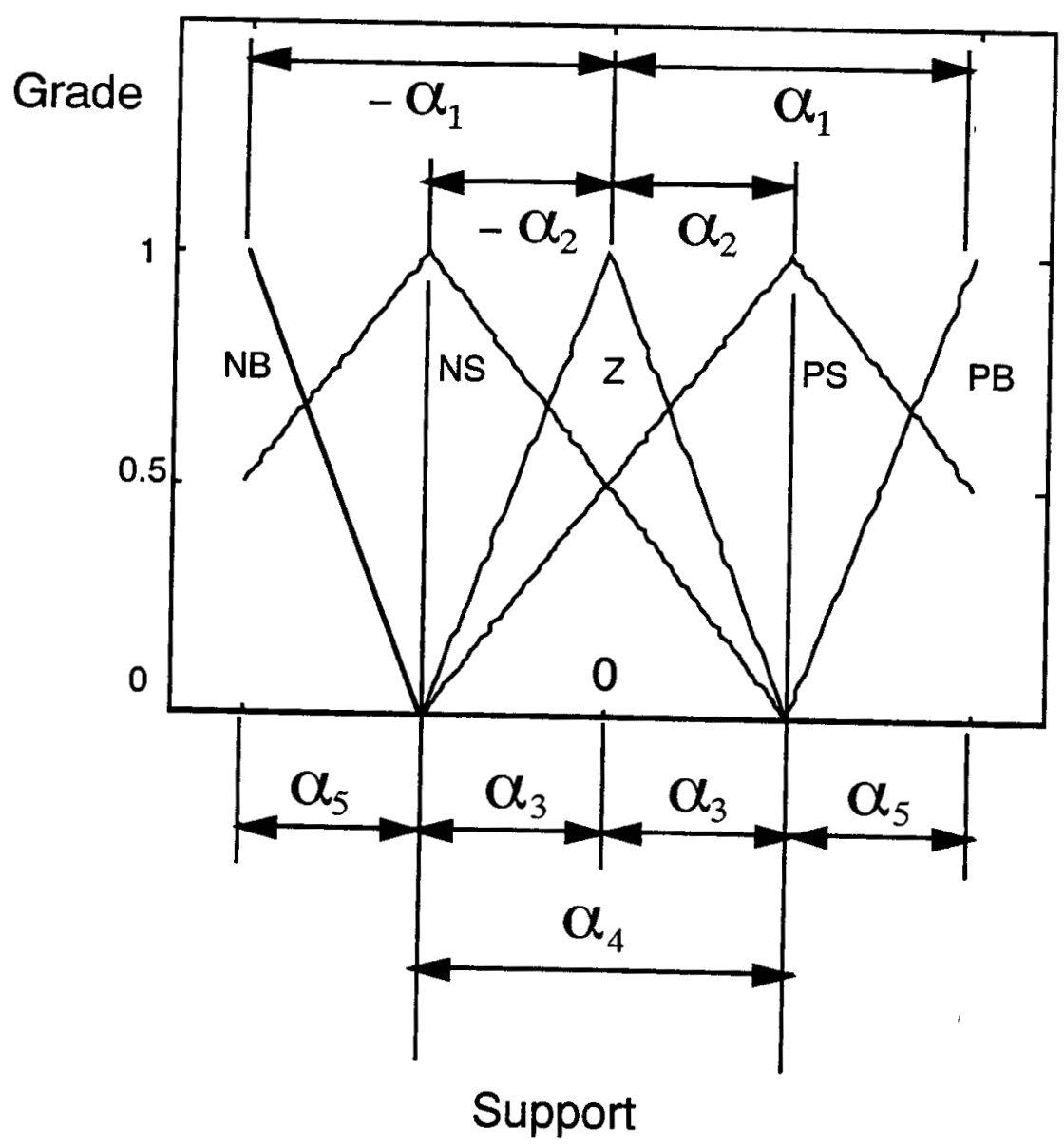

Fig. 5 Parameterized fuzzy membership function on a symmetric universe of discourse. 


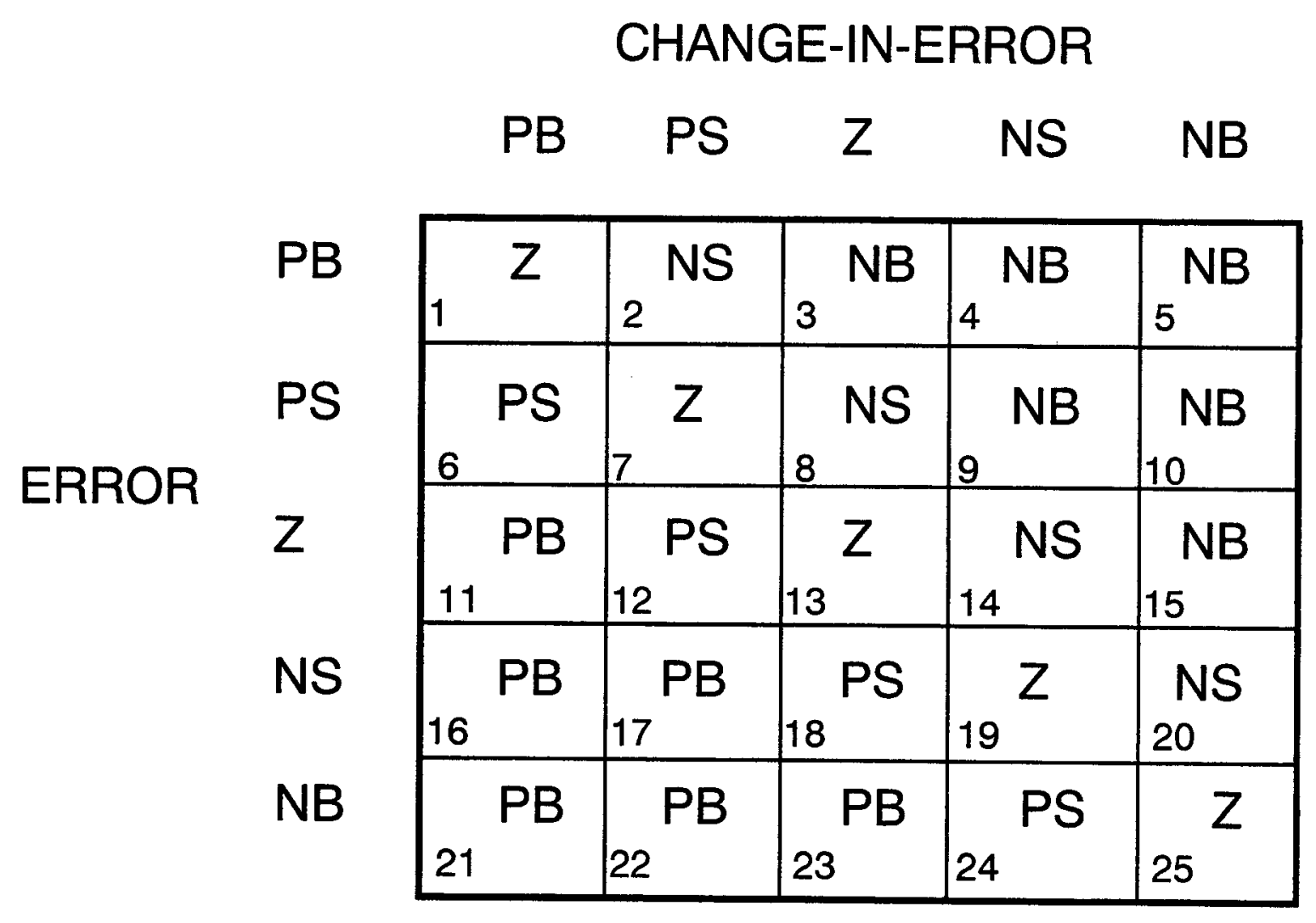

Fig. 6 Control rule matrix. 


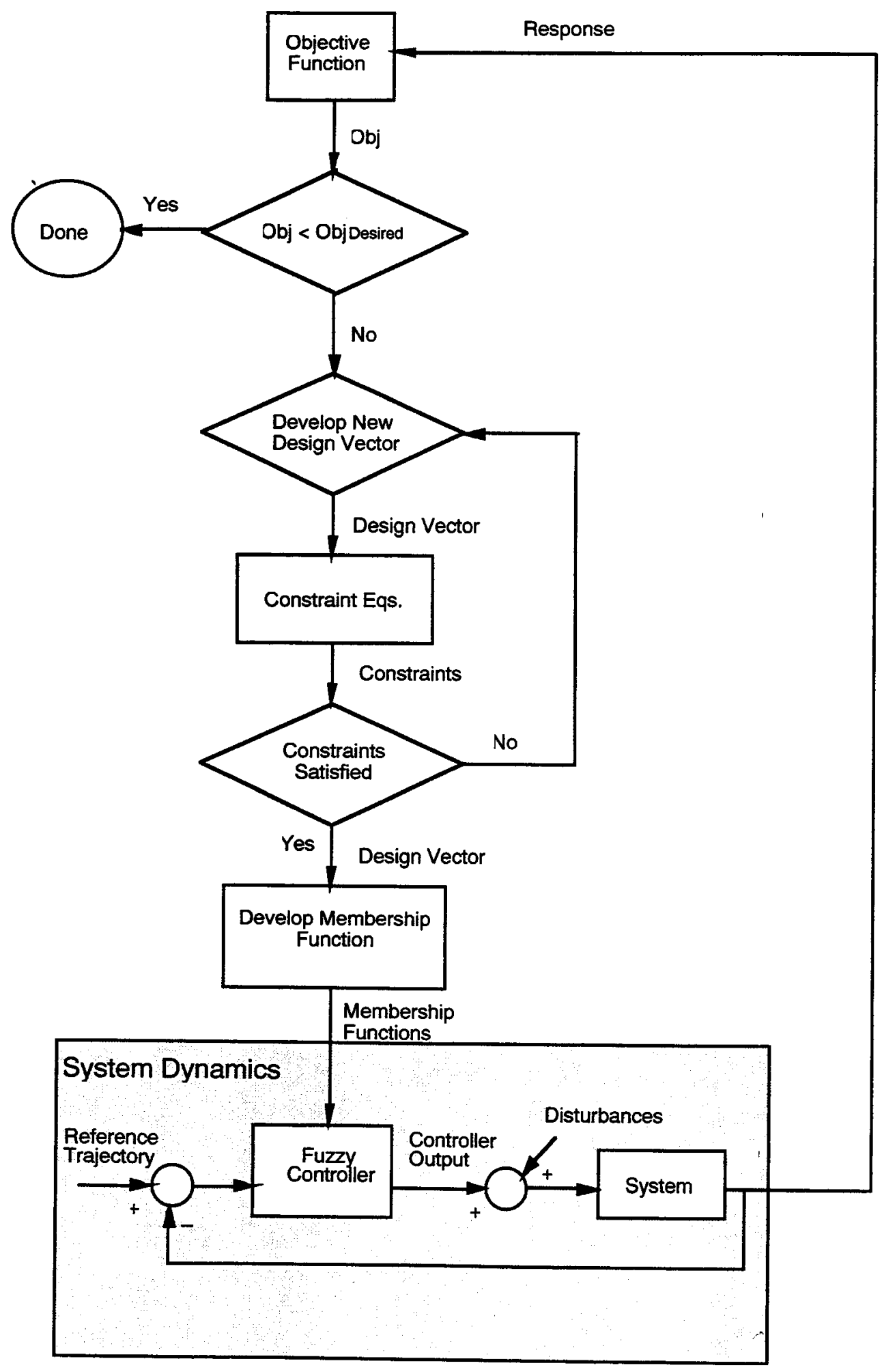

Fig. 7 Flowchart for tuning fuzzy controller. 


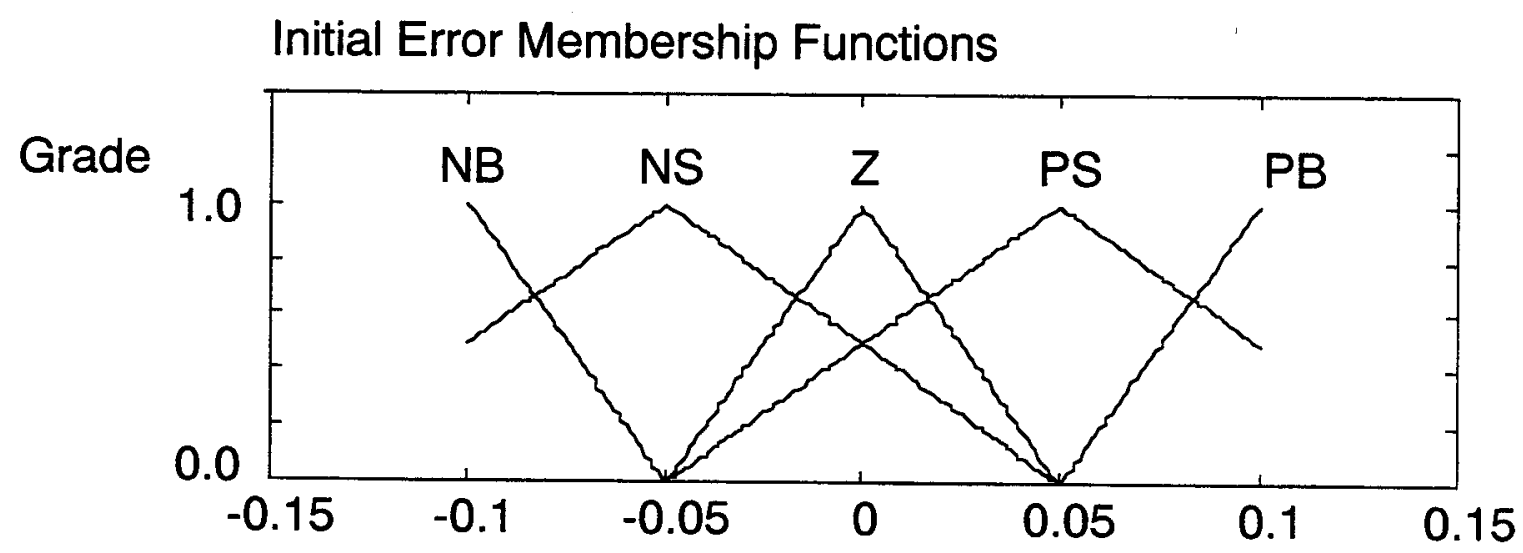

Final Error Membership Functions

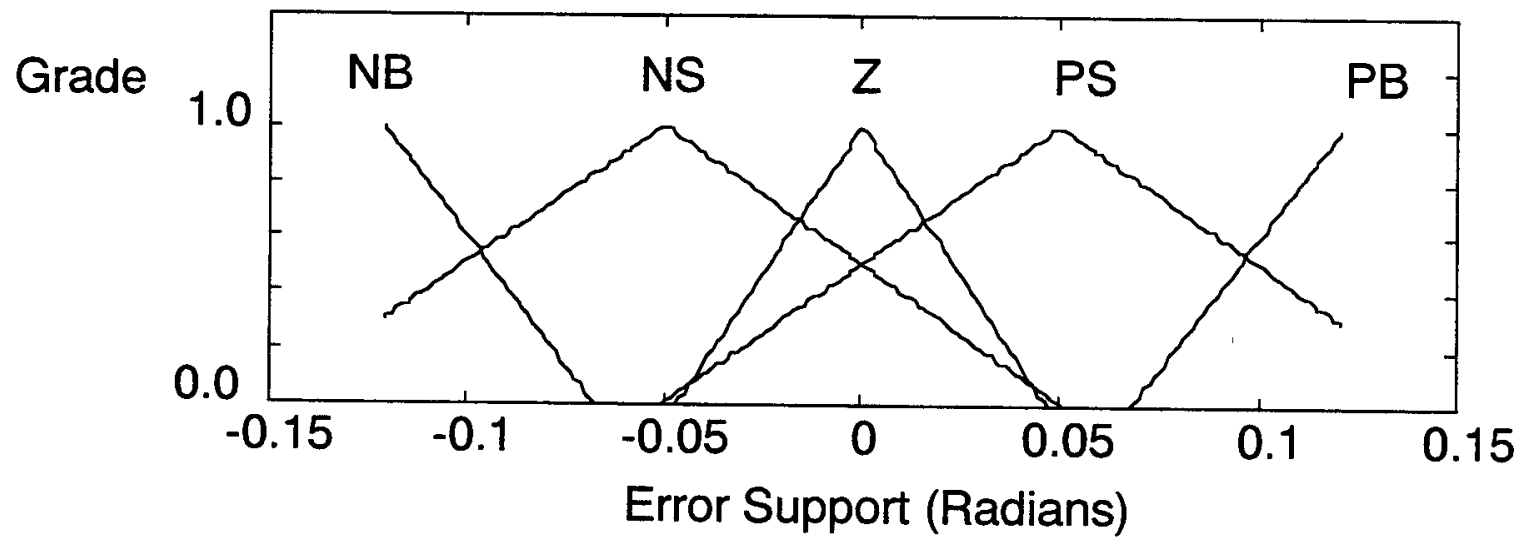

Fig. 8 Initial and final payload error membership function for slew maneuvers. 


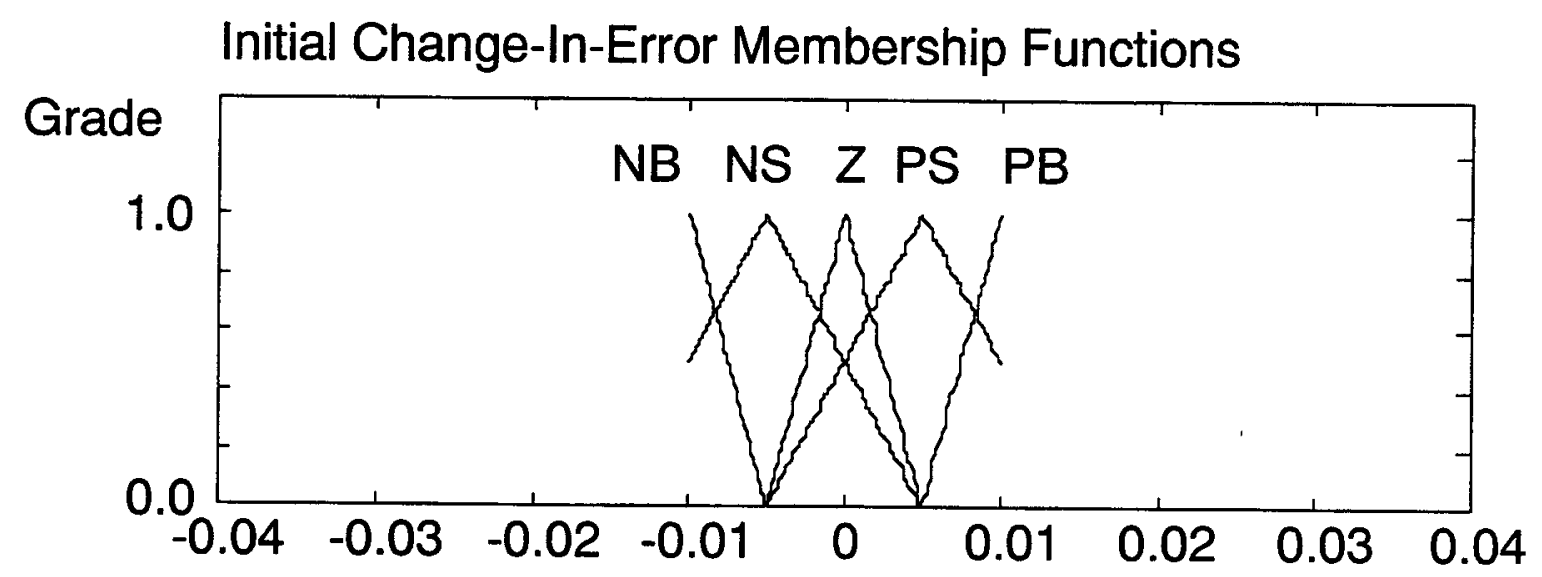

Final Change-In-Error Membership Functions

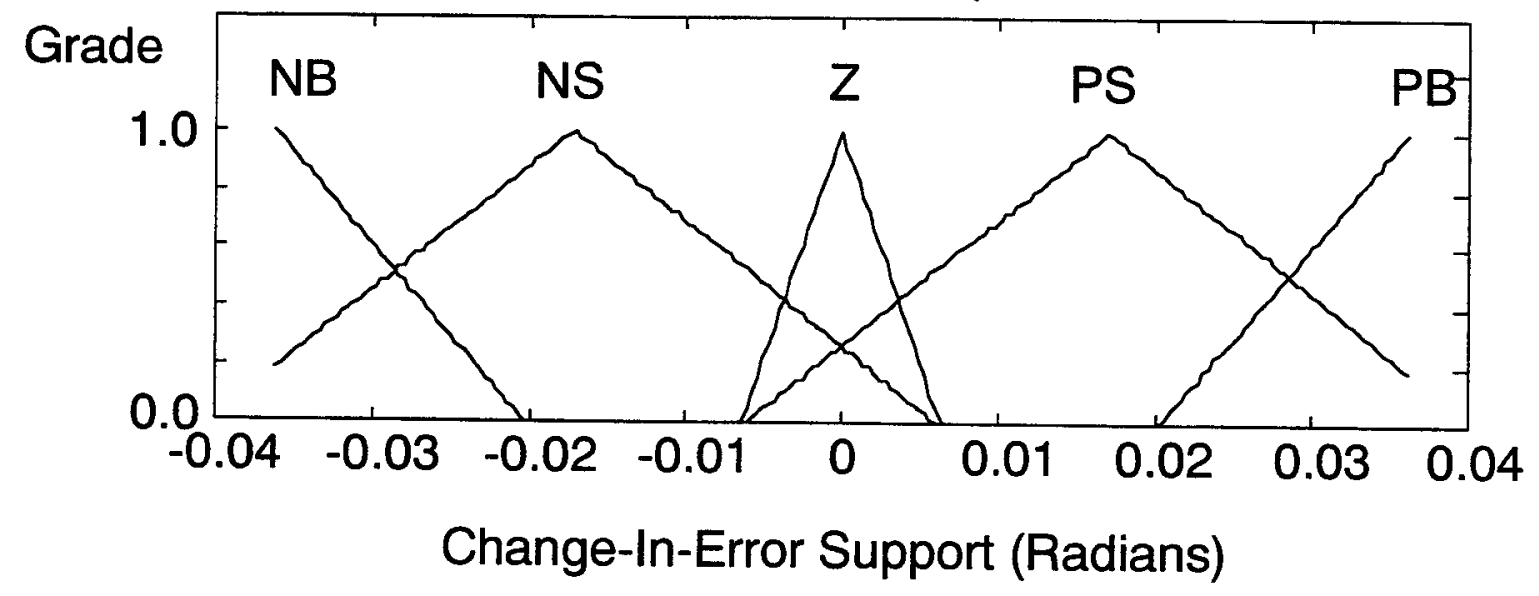

Fig. 9 Initial and final change-in-error membership function for slew maneuver. 


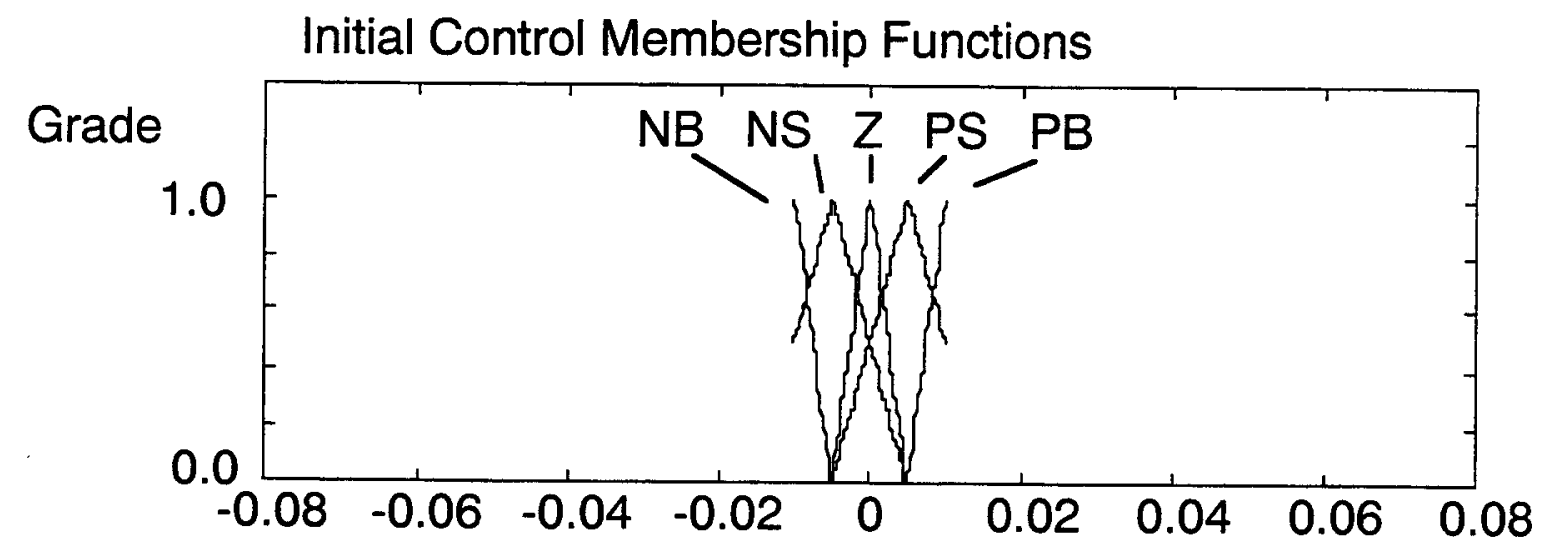

Final Control Membership Functions

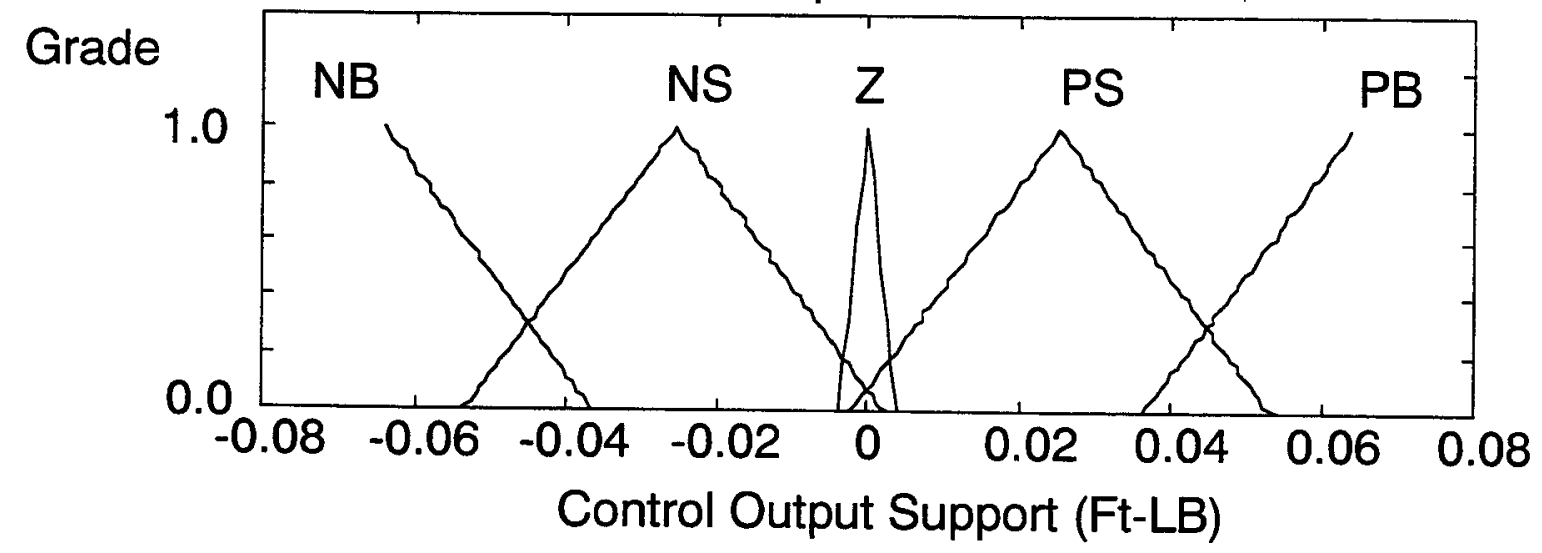

Fig. 10 Initial and final payload control membership function for slew maneuvers. 


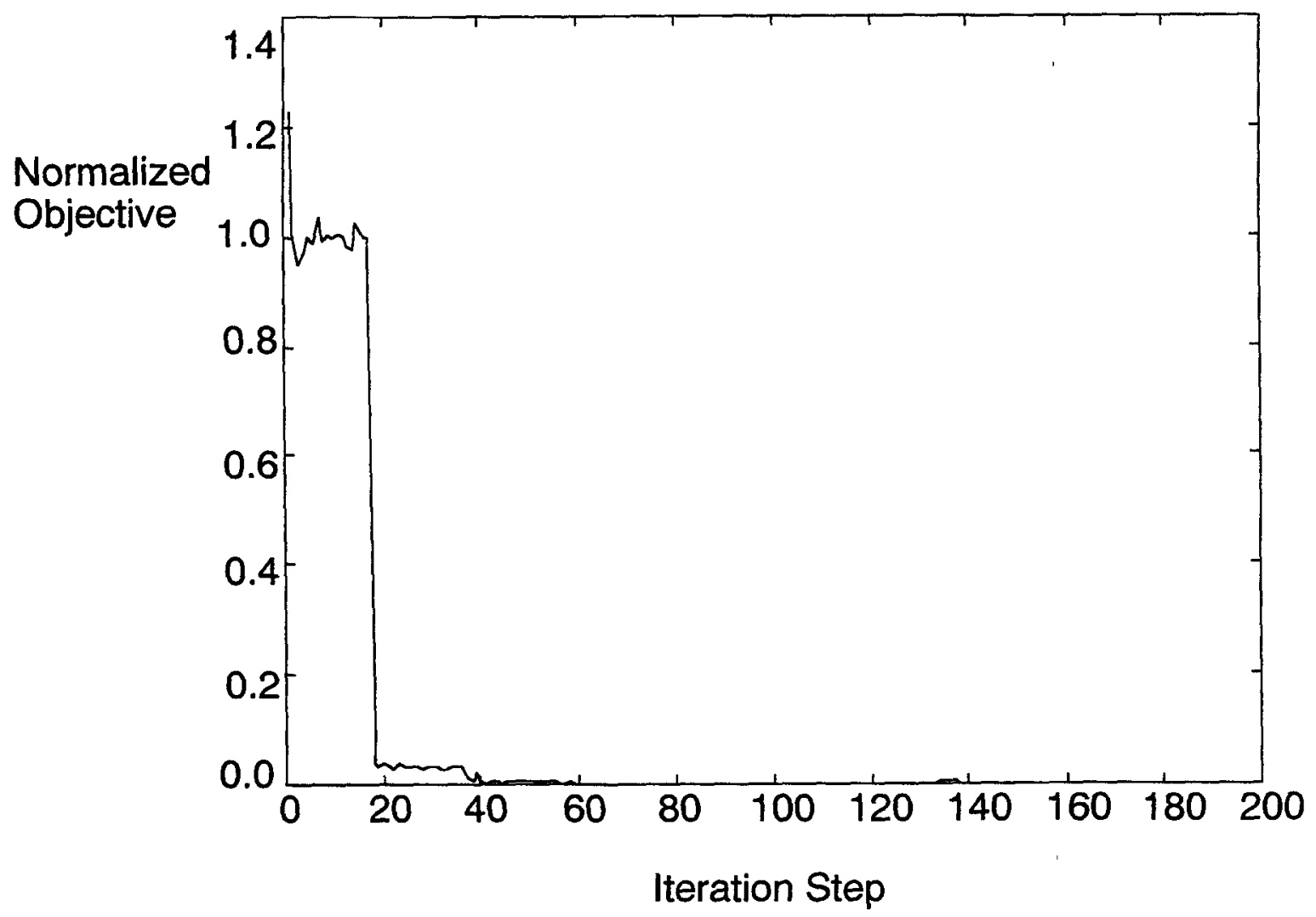

Fig. 11 Normalized payload objective iteration history resulting from tuning for slew maneuver. 


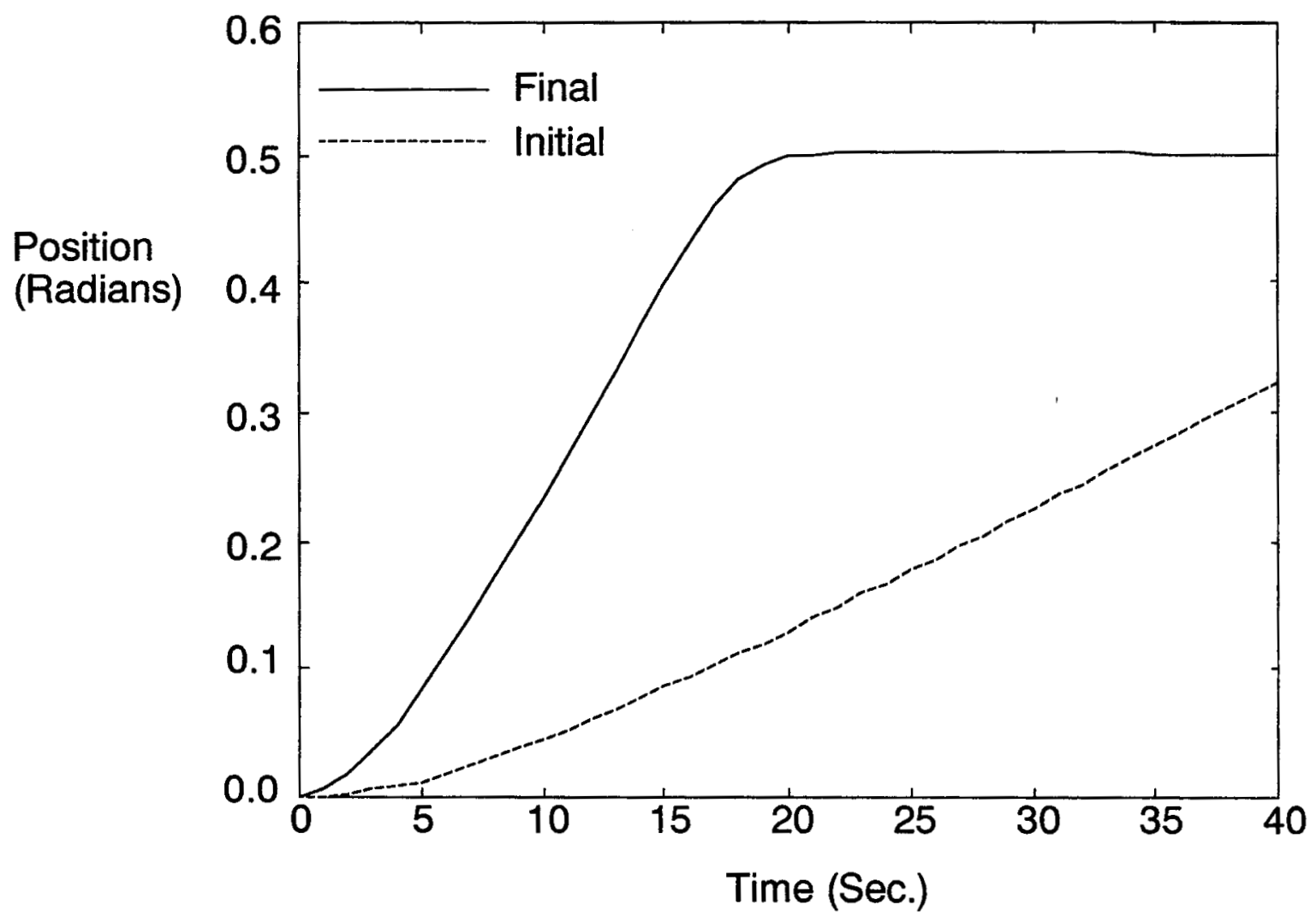

Fig. 12 Payload response to a commanded slew of $0.5 \mathrm{rad}$. using initial and final membership functions. 


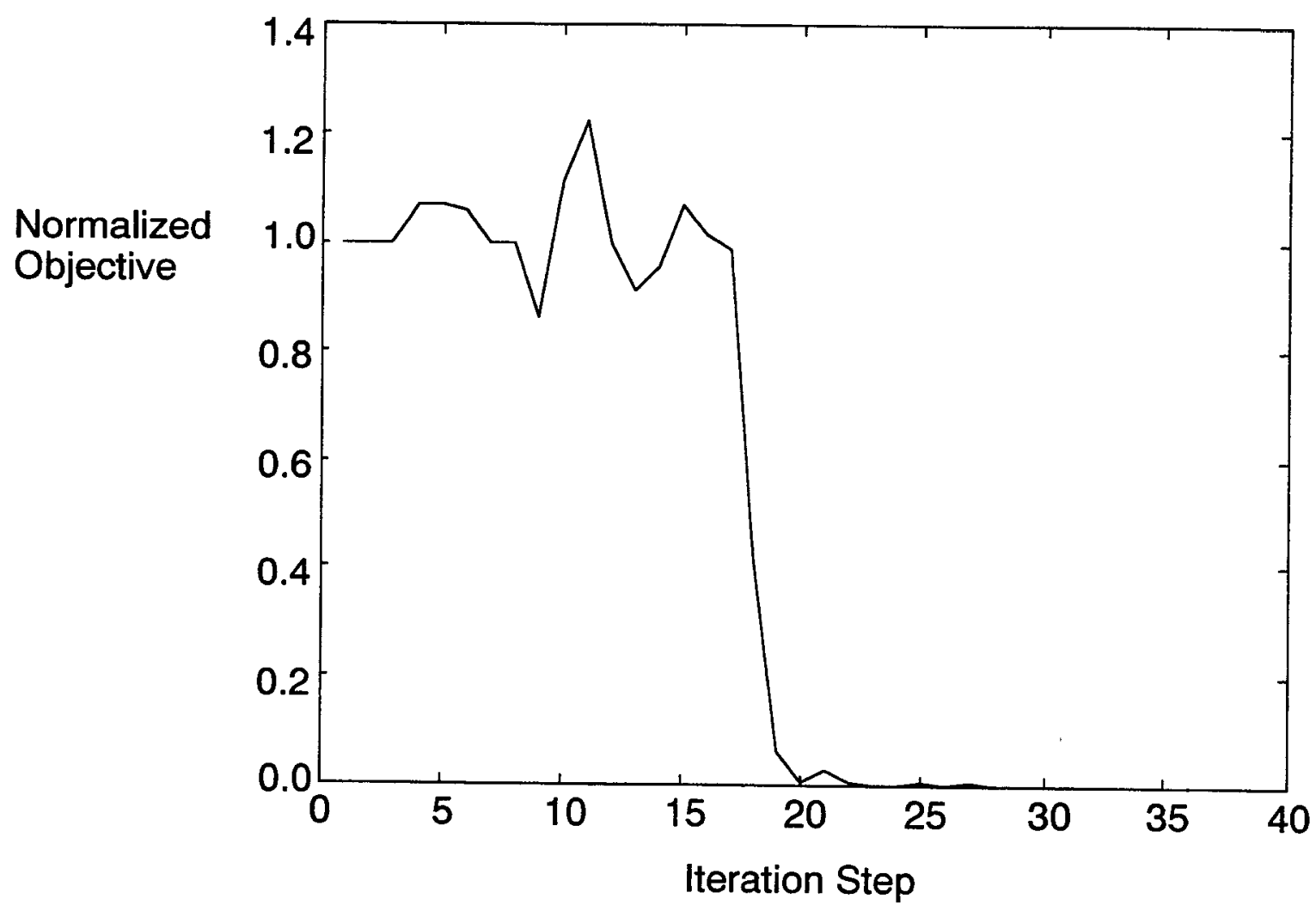

Fig. 13 Normalized payload objective iteration history while tuning for periodic disturbance rejection (0.01 ft-lb., $0.25 \mathrm{~Hz}$.) 


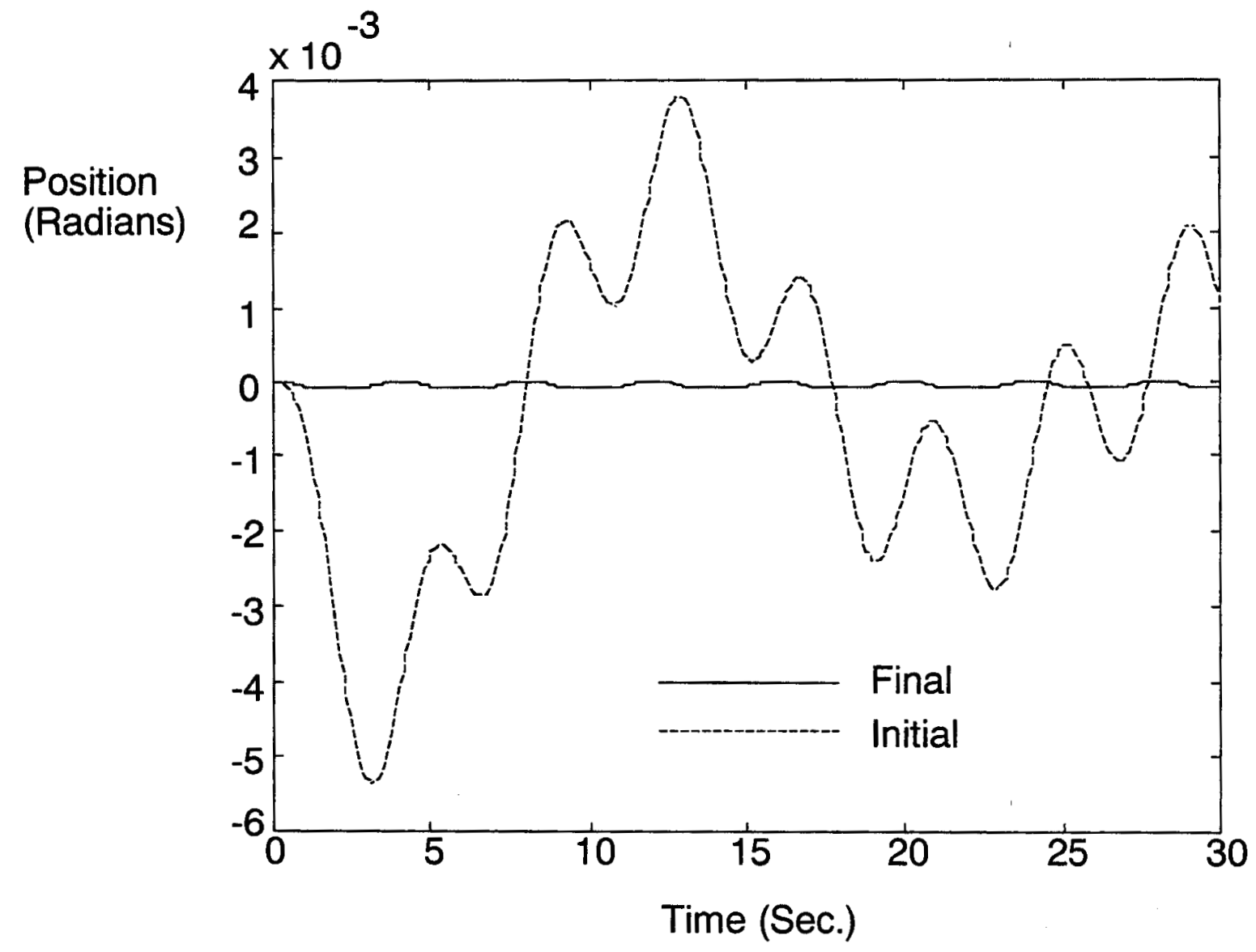

Fig. 14 Payload response to a periodic disturbance $(0.01 \mathrm{ft}-\mathrm{lb}, 0.25 \mathrm{~Hz})$ using initial and final membership functions. 